\title{
Structural analysis of brain ganglioside acetylation patterns in mice with altered ganglioside biosynthesis
}

\author{
Kristina Mlinac, Dragana Fabris, Željka Vukelić , Marko Rožman, Marija Heffer, Svjetlana Kalanj Bognar
}

\section{A B S T R A C T}

Gangliosides are sialylated membrane glycosphingolipids especially abundant in mammalian brain tis-sue. Sialic acid O-acetylation is one of the most common structural modifications of gangliosides which considerably influences their chemical properties. In this study, gangliosides extracted from brain tissue of mice with altered ganglioside biosynthesis (St8sia1 null and B4galnt1 null mice) were structurally characterized and their acetylation pattern was analyzed. Extracted native and alkali-treated gangliosides were resolved by high performance thin layer chromatography. Ganglioside mixtures as well as separated individual ganglioside fractions were further analyzed by tandem mass spectrometry. Several O-acetylated brain ganglioside species were found in knockout mice, not present in the wild-type mice. To the best of our knowledge this is the first report on the presence of O-acetylated GD1a in St8sial null mice and O-acetylated GM3 species in B4galnt1 null mice. In addition, much higher diver-sity of abnormally accumulated brain ganglioside species regarding the structure of ceramide portion was observed in knockout versus wild-type mice. Obtained findings indicate that the diversity of brain ganglioside structures as well as acetylation pat-terns in mice with altered ganglioside biosynthesis, is even higher than previously reported. Further investigation is needed in order to explore the effects of acetylation on ganglioside interactions with other molecules and consequently the physiological role of acetylated ganglioside species.

\section{Introduction}

Gangliosides are complex sialylated glycosphingolipids especially abundant and structurally diverse in mammalian nervous system. ${ }^{1}$ Gangliosides are anchored with their ceramide moiety in the outer leaflet of the plasma membrane, while the carbohydrate portion protrudes into the extracellular space. Therefore gangliosides interact with molecules (proteins as well as lipids) on adjacent cells and extracelullar matrix (trans interactions), as well as molecules within the same membrane (cis interactions)., Gangliosides are not evenly distributed throughout the membrane, but rather more concentrated in organized microdomains termed lipid rafts. ${ }^{4}$ Due to the complex structural properties, as well as unique location within the membrane, gangliosides are involved in multitude of processes including signaling events, apoptosis, proliferation, neurotransmitter positioning, pathogen binding, etc. ${ }^{2,3,5-9}$ It has been shown that specific brain ganglioside species participate in neuron-glia interactions, for example GD1a and GT1b are ligands for myelin-associated glycoprotein (MAG). ${ }^{10}$ Therefore, shared effect of changes in ganglioside composition, ganglioside modifications, and presence/absence of specific ganglioside species may lead to alterations in cell metabolism. ${ }^{11-15}$

Studies using genetically modified mouse models with disrupted ganglioside biosynthetic pathway have greatly contributed to the present body of knowledge on specific ganglioside roles. ${ }^{16,17}$ Ganglioside synthesis includes a stepwise action of different glycosyl and sialyl transferases ${ }^{9,18,19}$ (Fig. 1). Mice with changed ganglioside composition were generated by selectively knocking out specific biosynthetic enzymes of that pathway, for example, St8sia1 null mice lack the enzyme GD3 synthase (EC 2.4.99.8) with consequent deficiency in $b$ - and $c$-series gangliosides, while B4galnt1 null mice lack all complex gangliosides accumulating just GM3 and GD3 due to deficiency in GM2/GD2 synthase (EC 2.4.1.92). Phenotype of these mouse models varies greatly, from mildly impaired 


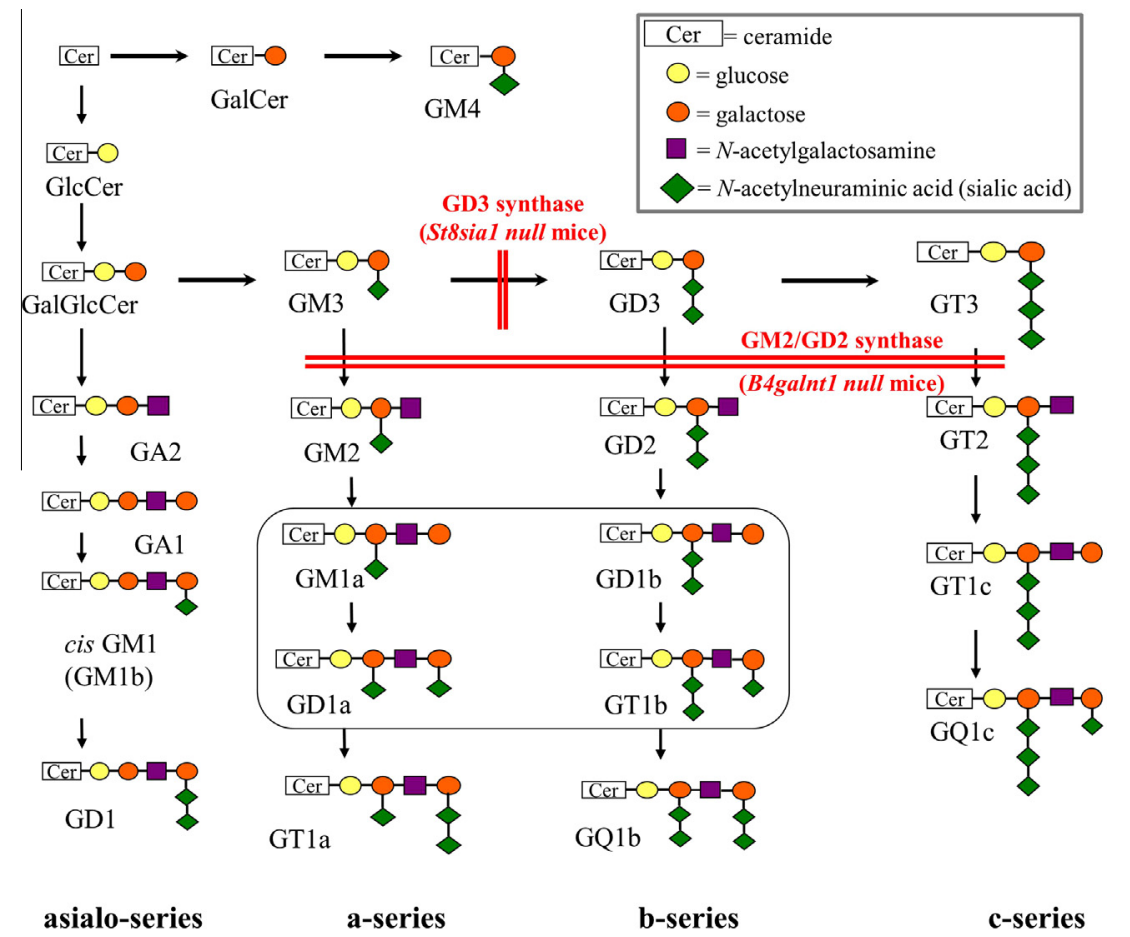

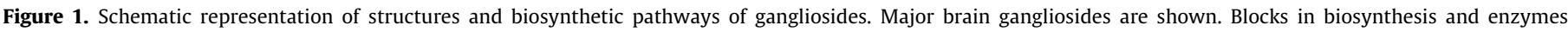
deficient in two genetically modified mouse models used in this study are indicated.

regeneration of lesioned nerves (St8sia1 null mice), to more severe manifestations which include demyelination, neurodegeneration, and motor deficits (B4galnt1 null mice). ${ }^{11,13,14,16,20-22}$ Even though the total concentration of gangliosides in the knockout (KO) mice remains the same as in the wild-type (wt) mice, the ganglioside composition is markedly changed since the loss of certain ganglioside species is compensated by overproduction of other gangliosides. Additional modifications have been reported for both ganglioside fractions present in normal ganglioside pattern as well as for the accumulated gangliosides. ${ }^{19,23,24} \boldsymbol{O}$-acetylation of sialic acid residues is one of the most common covalent modifications of gangliosides. Although acetylation is a relatively small structural change (Fig. 2), it has a considerable impact on physiological properties of gangliosides. Acetylation of sialic acids has been shown to serve as a protection against degradation by sialidases, influence receptor and pathogen binding, is considered a differentiation marker during development, and is implicated in tumor progression and spread. ${ }^{25-30}$

Even though ganglioside-deficient mice have been used in research for years, detailed structural analysis of all accumulated and modified gangliosides has not yet been fully investigated. Since acetylation can have a profound impact on ganglioside functions, the aim of this study was to investigate structural composition and the acetylation patterns of accumulated brain ganglioside species in two genetically modified mouse models, St8sia1 mull and B4galnt1 null mice.

\section{Results and discussion}

\subsection{Quantitative and qualitative analysis of native and alkali- treated ganglioside mixtures isolated from wt, St8sia1 null and B4galnt1 null mouse brain}

The total content of ganglioside-bound sialic acid (GG-SA) in brain tissue samples of St8sia1 null and B4galnt1 null mice did not significantly differ from wt mice $(378 \pm 69.12 \mu \mathrm{g} / \mathrm{g}$ and
$544.03 \pm 132.07 \mu \mathrm{g} / \mathrm{g}$ vs $504.44 \pm 69.12 \mu \mathrm{g} / \mathrm{g}$ ). The obtained values are in accordance to those previously reported. ${ }^{16,17}$ HPTLC separation of individual ganglioside species (Fig. 3) and their relative quantification by ImageJ analysis software confirmed ganglioside compositional differences typical for analyzed KO mice compared to wt mice (Table 1). ${ }^{16,17,19}$ However, comparison of composition of native and alkali-treated ganglioside mixtures from wt mice brain tissue indicated presence of additional alkali-labile ganglioside fractions (Fig. 3). A fraction which migrates directly above GT1b fraction could correspond to $O-A c-G Q 1 b$, since the relative proportion of GQ1b fraction was elevated after the alkaline treatment (Table 1). The second fraction migrates directly below GD1b fraction, and according to its $R_{\mathrm{f}}$ value it corresponds to previously reported $O$-Ac-GT1b. ${ }^{19}$ The third fraction migrating below GM1 was recognized as alkali-labile fraction since it completely disappeared upon alkaline treatment (Fig. 3). The fraction was attributed as O-Ac-GD1a, based on MS identification (Fig. 4, Table 2). However, the proportion of GD1a did not increase upon alkaline treatment which is explained by previously observed HPTLC co-migration of $O-A c-G D 1 b$ and GD1a. ${ }^{19}$ Thus the alkaline treatment led to slight decrease in GD1a proportion while increase in GD1b proportion was indeed recorded (Table 1). The fourth alkali-labile fraction was noticed within GD3-migrating zone, since the relative proportion of that zone was considerably reduced after the alkaline treatment (from $4.1 \%$ to $<1 \%$, Table 1 ).

The compositional analysis of St8sia1 null mice brain ganglioside mixture by HPTLC showed the presence of three ganglioside fractions. Two of them, GM1 and GD1a fractions, were expected considering the GD3 synthase deficiency and consequent absence of $b$-series gangliosides. The third fraction migrated below GM1 zone and was the only one which disappeared upon the alkaline treatment (Fig. 3, St8sia1 null $_{\mathrm{AH}}$ ). There are no available data on detailed structural analysis of St8sia1 null mice brain gangliosides, possibly due to limited interest because of relatively mild phenotype compared to other ganglioside-deficient mouse models. ${ }^{13,20}$ However, investigation of changed acetylation patterns in accumulated 


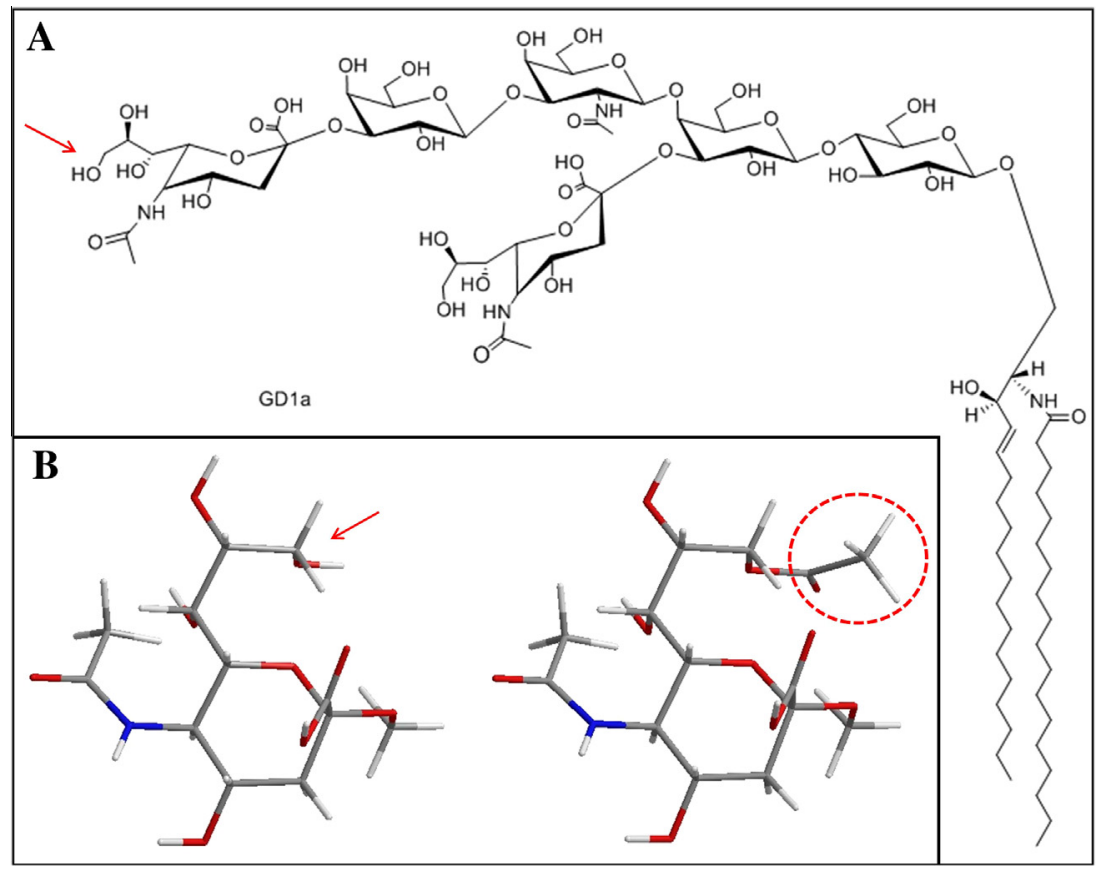

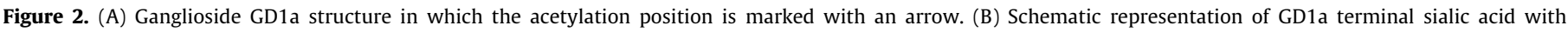
acetylation position marked with an arrow (left) and 9-O-acetylated sialic acid (right) conformations. Acetyl group of the outer sialic acid is circled.

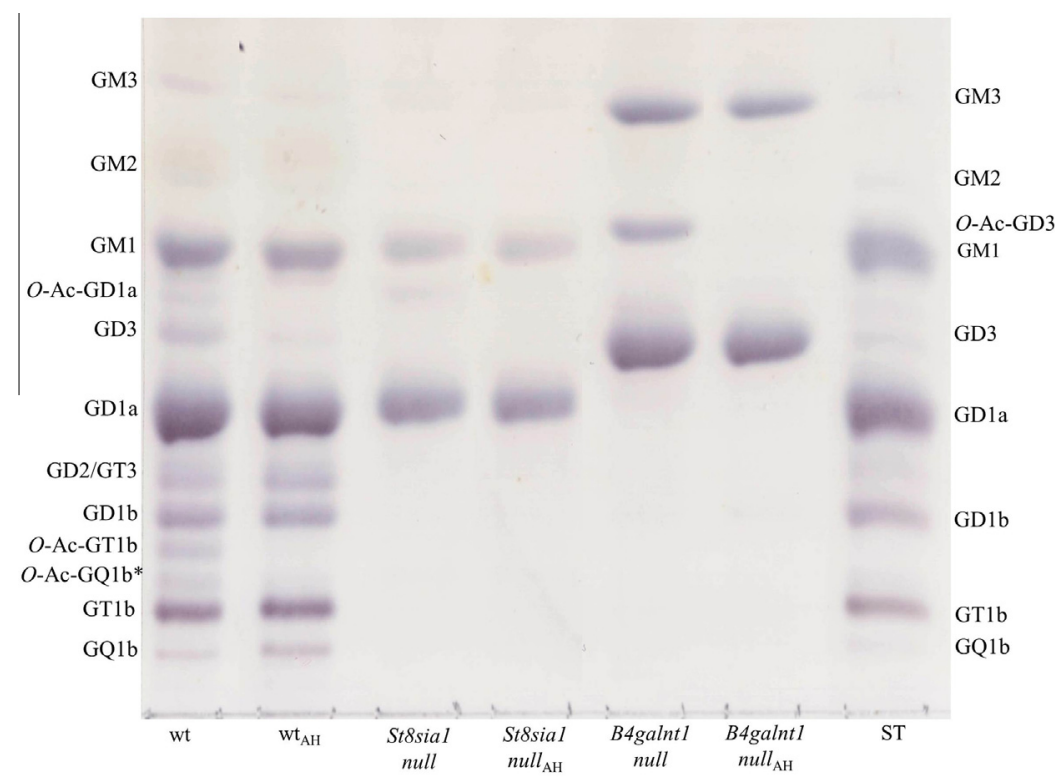

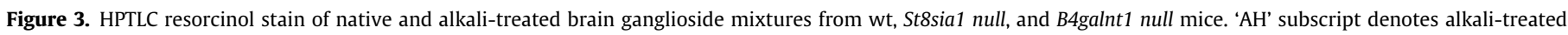
samples. *marks presumed $O$-Ac-GQ1b.

ganglioside species could provide a valuable insight into the probable compensation mechanisms which arise from dysfunctional ganglioside biosynthesis. Therefore we performed the analysis of this alkali-labile ganglioside species by mass spectrometry.

The HPTLC analysis of B4galnt1 null mice brain gangliosides showed three major fractions corresponding to GD3, $O$-acetylated GD3, and GM3, in accordance to the GM2/GD2 synthase deficiency and consequent depletion of all complex ganglioside structures. The $O$-Ac-GD3 migrating fraction disappeared upon the alkaline treatment (Fig. 3, B4galnt1 null ${ }_{\mathrm{AH}}$ ). The acetylation of GD3, the fraction heavily accumulated in GM2/GD2 synthase-deficient mice, was previously confirmed by immunohistochemical localization and mass spectrometry in studies by Matsuda et al. ${ }^{23}$ and Furukawa et al. ${ }^{24}$ We did not detect $O-A c-G D 3$ in wt mouse brain tissue since it is a very minor ganglioside in the adult brain, while in developing brain, the expression of $O-A c-G D 3$ has been associated to processes such as neurite outgrowth and neuronal migration in mammalian brain. ${ }^{31-33}$ Interestingly, it was found that exogenously added GD3 induces its own $O$-acetylation machinery in different cell types, ${ }^{34}$ while other gangliosides fail to induce the same machinery. This finding implies that $O$-acetylation machinery requires very specific oligosaccharide moieties of gangliosides and that not all ganglioside species are susceptible to $O$-acetylation in the same proportion. 
Table 1

Relative proportions of individual ganglioside species present in total native and alkali-treated ganglioside mixtures isolated from wild-type, St8sia1 null, and B4galnt1 null mouse brain tissue

\begin{tabular}{|c|c|c|c|c|c|c|}
\hline \multirow[t]{2}{*}{ Ganglioside } & \multicolumn{6}{|c|}{ Proportion of individual ganglioside (\%) } \\
\hline & wt & $\mathrm{wt}_{\mathrm{AH}}$ & St8sia1 null & St8sia1 null $_{\mathrm{AH}}$ & B4galnt1 null & B4galnt1 null $\mathrm{AH}_{\mathrm{H}}$ \\
\hline GM3 & $<1$ & $<1$ & 2.2 & 2.2 & 28.2 & 28.5 \\
\hline GM2 & $<1$ & $<1$ & 1.6 & 1.5 & - & - \\
\hline O-Ac-GD3 & - & - & - & - & 16 & - \\
\hline GM1 & 19.9 & 18.6 & 20.6 & 20.1 & - & - \\
\hline O-Ac-GD1a & $<1$ & - & 4.9 & - & - & - \\
\hline GD3 & 4.1 & $<1$ & - & - & 55.8 & 71.5 \\
\hline GD1a/(O-Ac-GD1b) & 44.1 & 42.4 & 70.7 & 76.2 & - & - \\
\hline GD2/GT3 & 4.3 & 5 & - & - & - & - \\
\hline GD1b & 8.1 & 9.2 & - & - & - & - \\
\hline O-Ac-GT1b & 2.2 & - & - & - & - & - \\
\hline O-Ac-GQ1b* & $<1$ & - & - & - & - & - \\
\hline GT1b & 13.2 & 19.5 & - & - & - & - \\
\hline GQ1b & 1.3 & 3.7 & - & - & - & - \\
\hline
\end{tabular}

wt, wild-type; ‘AH’ subscript denotes alkali-treated samples; -, not detected; *presumed O-Ac-GQ1b.

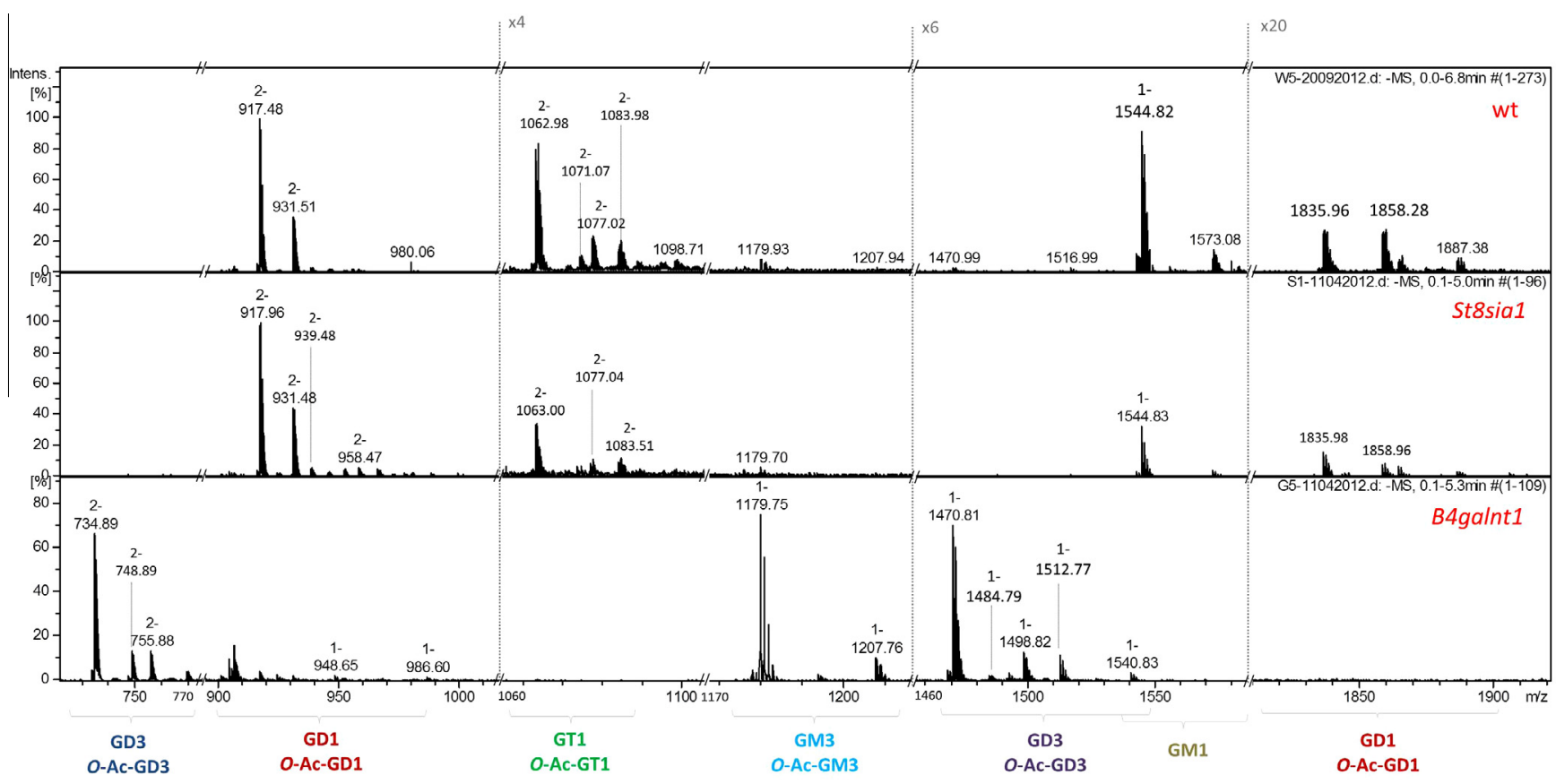

Figure 4. Mass spectra of native ganglioside mixtures from wt, St8sia1 null, and B4galnt1 null mouse brain samples.

\subsection{Mass spectrometric analysis of native ganglioside mixtures isolated from wt, St8sia1 null and B4galnt1 null mouse brain tissue}

The purified native ganglioside mixtures isolated from wt, St8sia1 null and B4galnt1 null mice brains were directly screened by electrospray ionization mass spectrometry (ESI MS). The obtained spectra (MS1) are presented in Figure 4, and mass region containing the ions with lower abundance is shown in Supplementary Figure 1. A complex molecular ion pattern was detected, confirming corresponding chromatographically visualized ganglioside patterns. All major individual ganglioside fractions showed high diversity in their ceramide portions. The only species that was beyond the detection limit was GQ1b. Detected molecular ions and their corresponding proposed ganglioside structures are presented in Table 2.

The spectrum of wt mice brain ganglioside mixture was the most complex of the analyzed samples showing all of the ganglioside species typically present in mice brain.
In the spectrum of St8sia1 null mice brain ganglioside mixture, the ions corresponding to GD3 gangliosides are missing. The most abundant signals in MS scans are those corresponding to GD1 and GM1 species which is in accordance to HPTLC data. The ions corresponding to GT1 (d18:1/18:0) and GT1 (18:1/20:0) (doubly charged ion at $m / z$ 1077.02) ganglioside species were also detected although GT1 fraction was not observed on HPTLC due to its much lower sensitivity, as well as rather low amount of ganglioside mixture applied to the plate. GT1 ganglioside species were further subjected to structural analysis by MS/MS sequencing in the negative ion mode. The product ion spectrum of the doubly charged molecular ion at $m / z$ 1063.00, corresponding to GT1 (d18:1/18:0) is shown in Figure 5. The spectrum confirms the presence of trisialylated tetraose structure which may be attributed to GT1a isomer since the biosynthetic pathway of $b$ - and $c$-series gangliosides is blocked in this KO model.

B4galnt1 null mouse brain ganglioside mixture spectrum (Fig. 4) shows abundant ions that correspond to GM3, O-acetyl-GM3, GD3, and $O$-acetyl-GD3 species. These species accumulated in B4galnt1 
Table 2

The most abundant ganglioside molecular ions detected by mass spectrometric screening of native ganglioside mixtures isolated from brain tissue of wild-type, St8sia1null, and B4galnt1 null mice, and their corresponding proposed ganglioside structures

\begin{tabular}{|c|c|c|c|c|c|}
\hline $\mathrm{m} / \mathrm{z}$ & Molecular ion & Proposed structure & WT & St & B4 \\
\hline 1179.75 & {$[\mathrm{M}-\mathrm{H}]^{-}$} & GM3 (d18:1/18:0) & + & + & + \\
\hline 1193.72 & {$[\mathrm{M}-\mathrm{H}]^{-}$} & GM3 (d18:1/19:0) & & & + \\
\hline 1207.76 & {$[\mathrm{M}-\mathrm{H}]^{-}$} & GM3 (d18:1/20:0) & + & & + \\
\hline 1221.75 & {$[\mathrm{M}-\mathrm{H}]^{-}$} & O-Ac-GM3 (d18:1/18:0) & + & & + \\
\hline 1235.75 & {$[\mathrm{M}-\mathrm{H}]^{-}$} & GM3 (d18:1/22:0) & & & + \\
\hline 1257.88 & {$[\mathrm{M}+\mathrm{Na}-2 \mathrm{H}]^{-}$} & & + & & \\
\hline 1261.71 & {$[\mathrm{M}-\mathrm{H}]^{-}$} & GM3 (d18:1/24:1) & & & + \\
\hline 1299.68 & {$[\mathrm{M}+\mathrm{Na}-2 \mathrm{H}]^{-}$} & O-Ac-GM3 (d18:1/22:0) & & & + \\
\hline 734.89 & {$[\mathrm{M}-\mathrm{H}]^{-}$} & GD3 (d18:1/18:0) & & & + \\
\hline 1470.81 & {$[\mathrm{M}-\mathrm{H}]^{-}$} & & + & & + \\
\hline 1484.79 & {$[\mathrm{M}-\mathrm{H}]^{-}$} & GD3 (d18:1/19:0) & & & + \\
\hline 748.89 & {$[\mathrm{M}-2 \mathrm{H}]^{2-}$} & GD3 (d18:1/20:0) & & & + \\
\hline 1498.82 & {$[\mathrm{M}-\mathrm{H}]^{-}$} & & & & + \\
\hline 755.88 & {$[\mathrm{M}-2 \mathrm{H}]^{2-}$} & O-Ac-GD3 (d18:1/18:0) & & & + \\
\hline 1512.77 & {$[\mathrm{M}-\mathrm{H}]^{-}$} & & & & + \\
\hline 769.90 & {$[\mathrm{M}-2 \mathrm{H}]^{2-}$} & O-Ac-GD3 (d18:1/20:0) & & & + \\
\hline 1540.83 & {$[\mathrm{M}-\mathrm{H}]^{-}$} & & & & + \\
\hline 1516.99 & {$[\mathrm{M}-\mathrm{H}]^{-}$} & GM1 (d18:1/16:0) & + & + & \\
\hline 1544.82 & {$[\mathrm{M}-\mathrm{H}]^{-}$} & GM1 (d18:1/18:0) & + & + & \\
\hline 1572.98 & {$[\mathrm{M}-\mathrm{H}]^{-}$} & GM1 (d18:1/20:0) & + & + & \\
\hline 917.48 & {$[\mathrm{M}-2 \mathrm{H}]^{2-}$} & GD1 (d18:1/18:0) & + & + & \\
\hline 1835.96 & {$[\mathrm{M}-\mathrm{H}]^{-}$} & & + & + & \\
\hline 1858.28 & {$[\mathrm{M}+\mathrm{Na}-2 \mathrm{H}]^{-}$} & & + & + & \\
\hline 931.51 & {$[\mathrm{M}-2 \mathrm{H}]^{2-}$} & GD1 (d18:1/20:0) & + & + & \\
\hline 1885.96 & {$[\mathrm{M}+\mathrm{Na}-2 \mathrm{H}]^{-}$} & & + & + & \\
\hline 938.45 & {$[\mathrm{M}-2 \mathrm{H}]^{2-}$} & O-Ac-GD1 (d18:1/18:0) & + & + & \\
\hline 1877.91 & {$[\mathrm{M}-\mathrm{H}]^{-}$} & & + & + & \\
\hline 945.48 & {$[\mathrm{M}-2 \mathrm{H}]^{2-}$} & GD1 (d18:1/22:0) & + & + & \\
\hline 952.47 & {$[\mathrm{M}-2 \mathrm{H}]^{2-}$} & O-Ac-GD1 (d18:1/20:0) & + & + & \\
\hline 958.46 & {$[\mathrm{M}-2 \mathrm{H}]^{2-}$} & GD1 (d18:1/24:1) & + & + & \\
\hline 980.44 & {$[\mathrm{M}-2 \mathrm{H}]^{2-}$} & $O-A c-G D 1(\mathrm{~d} 18: 1 / 24: 0)$ & + & + & \\
\hline 1062.98 & {$[\mathrm{M}-2 \mathrm{H}]^{2-}$} & GT1 (d18:1/18:0) & + & & \\
\hline 1071.07 & {$[\mathrm{M}-2 \mathrm{H}]^{2-}$} & O-Ac-GT1 (d18:1/16:0) & + & & \\
\hline 1077.02 & {$[\mathrm{M}-2 \mathrm{H}]^{2-}$} & GT1 (d18:1/20:0) & + & + & \\
\hline 1083.98 & {$[\mathrm{M}-2 \mathrm{H}]^{2-}$} & O-Ac-GT1 (d18:1/18:0) & + & + & \\
\hline 1098.02 & {$[\mathrm{M}-2 \mathrm{H}]^{2-}$} & O-Ac-GT1 (d18:1/20:0) & + & & \\
\hline
\end{tabular}

Data on the most abundant ions are given, in accordance to MS scan presented in Figure 4.

null mice are present in minor to neglectable quantities in wt mice. All four ganglioside fractions from B4galnt1 null brain tissue showed high diversity of ganglioside species regarding the structure of ceramide portion (Supplementary Table 1 ). $O$-acetylated GM3 species was not previously reported in a murine model with disrupted ganglioside biosynthesis.
2.3. Mass spectrometric analysis of preparatively isolated GD1amigrating fractions from wt and St8sia1 null mouse brain ganglioside mixtures

Gangliosides of $b$-series are absent in St8sia1 null mice brain while GM1 and GD1a gangliosides accumulate. ${ }^{13,20}$ In order to compare the GD1a composition of St8sia1 null versus wt mice brain, both HPTLC GD1a-migrating fractions were isolated and analyzed by MS. The MS screening revealed numerous signals in GD1a-migrating fractions of both samples (Fig. 6). Keeping in mind that $O$-acetylated GD1b species co-migrate with GD1a species, ${ }^{19}$ it is likely that $O$-acetylated GD1b species were present in wt mice GD1a-migrating fraction, while in St8sia1 null mice brain only GD1a species were present in the analyzed fraction. The GD1amigrating fraction total ion intensity was higher in St8sia1 null than in wt mice brain, which is in accordance with the accumulation of GD1a species due to GD3 synthase deficiency in St8sia1 null mice. Since the $m / z$ values of some $O$-Ac-GD1b and GD1a gangliosides are very close (doubly charged ions at $m / z 938.48$ for $O$-Ac-GD1 (d18:1/18:0), 938.50 for GD1 (d18:1/21:0) and 939.48 for GD1 (t18:1/20:0)), we suppose that more than one ion was present within the same peak. Higher abundance of $O$-acetylated GD1a species was detected by MS1 in St8sia1 null vs. wt mice brain, as observed by HPTLC analysis. Interestingly, a higher abundance of $O$-acetylated GD1 (GD1b) has been reported for St3gal2/3 double null mice. ${ }^{19}$ It seems that total acetylation of gangliosides is maintained in both wt and KO murine brain tissue, e.g. observed increased proportions of acetylated ganglioside species in described KO-models were a consequence of different distribution of acetylation, depending on the type of block in ganglioside biosynthesis. Specific effects, interactions, and physiological roles of (accumulated) acetylated gangliosides have yet to be explored.

\subsection{Mass spectrometric and tandem mass spectrometric analysis of preparatively isolated ganglioside fraction from St8sia1 null mouse brain containing GM1 and 0 -Ac-GD1a}

The two ganglioside fractions from St8sia1 null mice brain observed on HPTLC migrating as GM1 standard and presumed $O$-Ac-GD1a (Fig. 3) were scrapped from the plate and preparatively isolated in order to perform more detailed structural analysis. Figure 7 represents the spectrum of that fraction obtained by MS1 screening; the molecular ions corresponding to GM1 and O-AcGD1 species are also represented. MS analysis revealed several ions corresponding to GM1 ganglioside species with different ceramide moieties, together with signals that correspond to $O$-acetylated

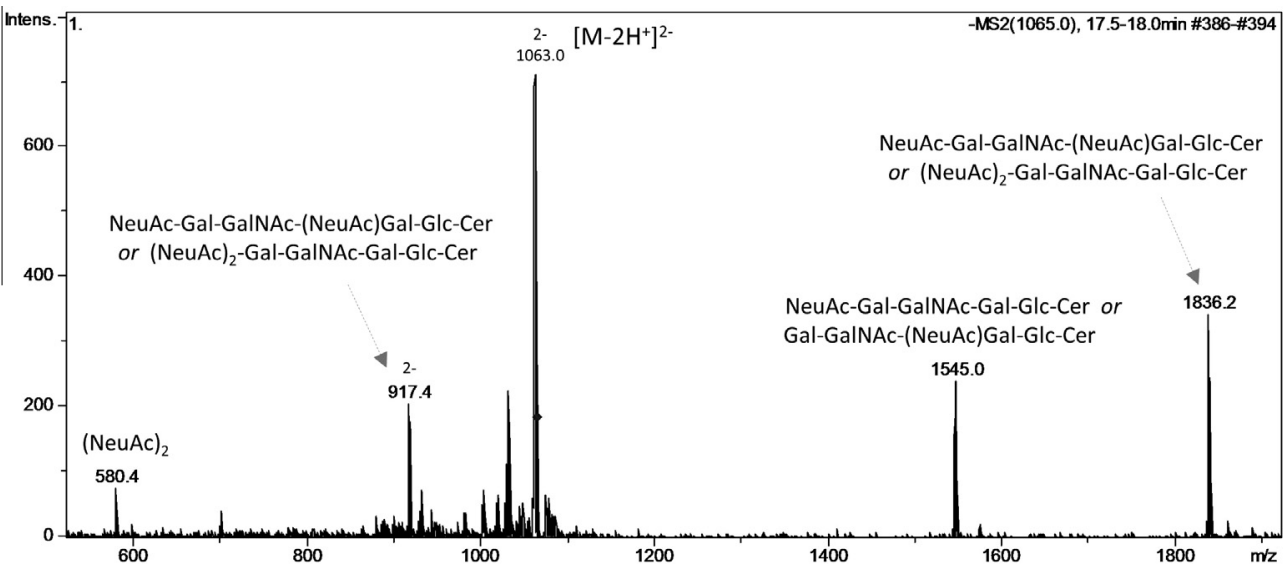

Figure 5. The product ion spectrum of the doubly charged molecular ion at $m / z 1063.00$, corresponding to GT1a (d18:1/18:0). 


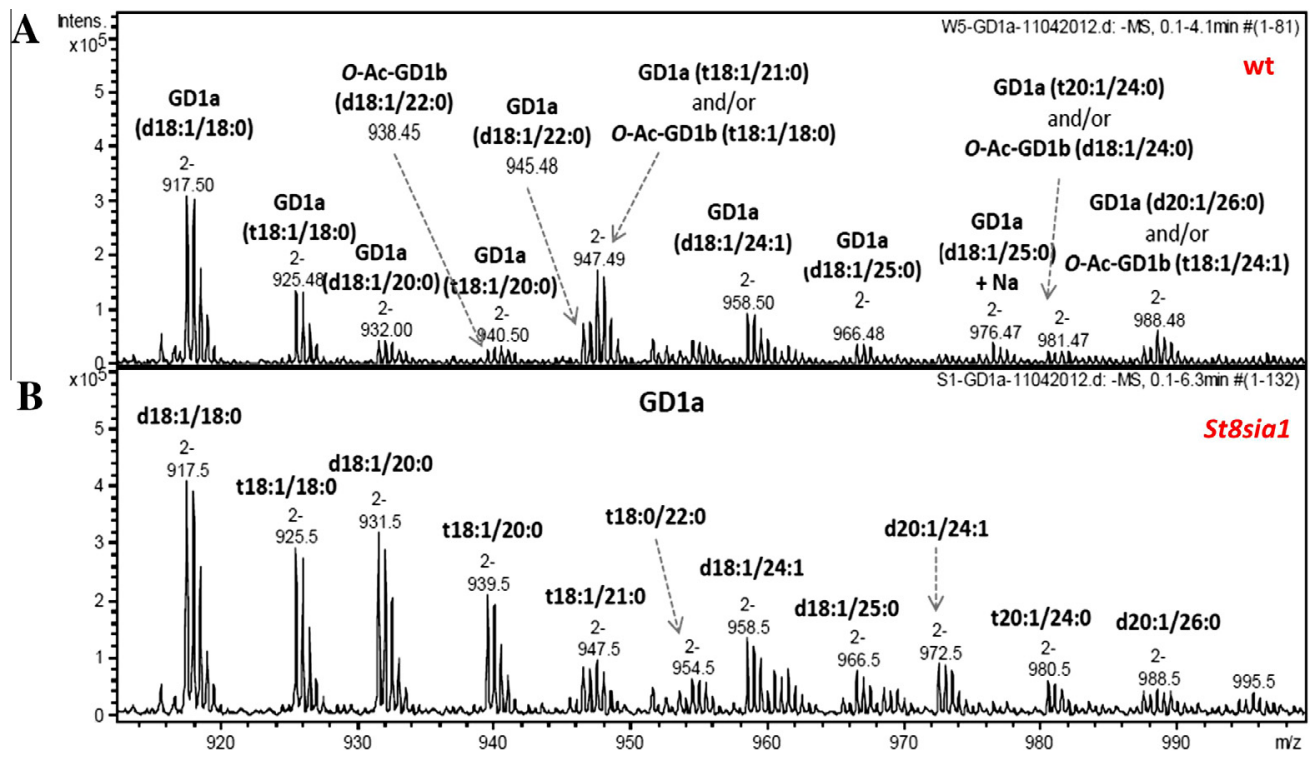

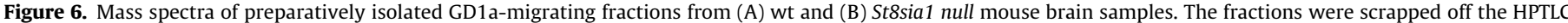
plate and extracted from silica gel.

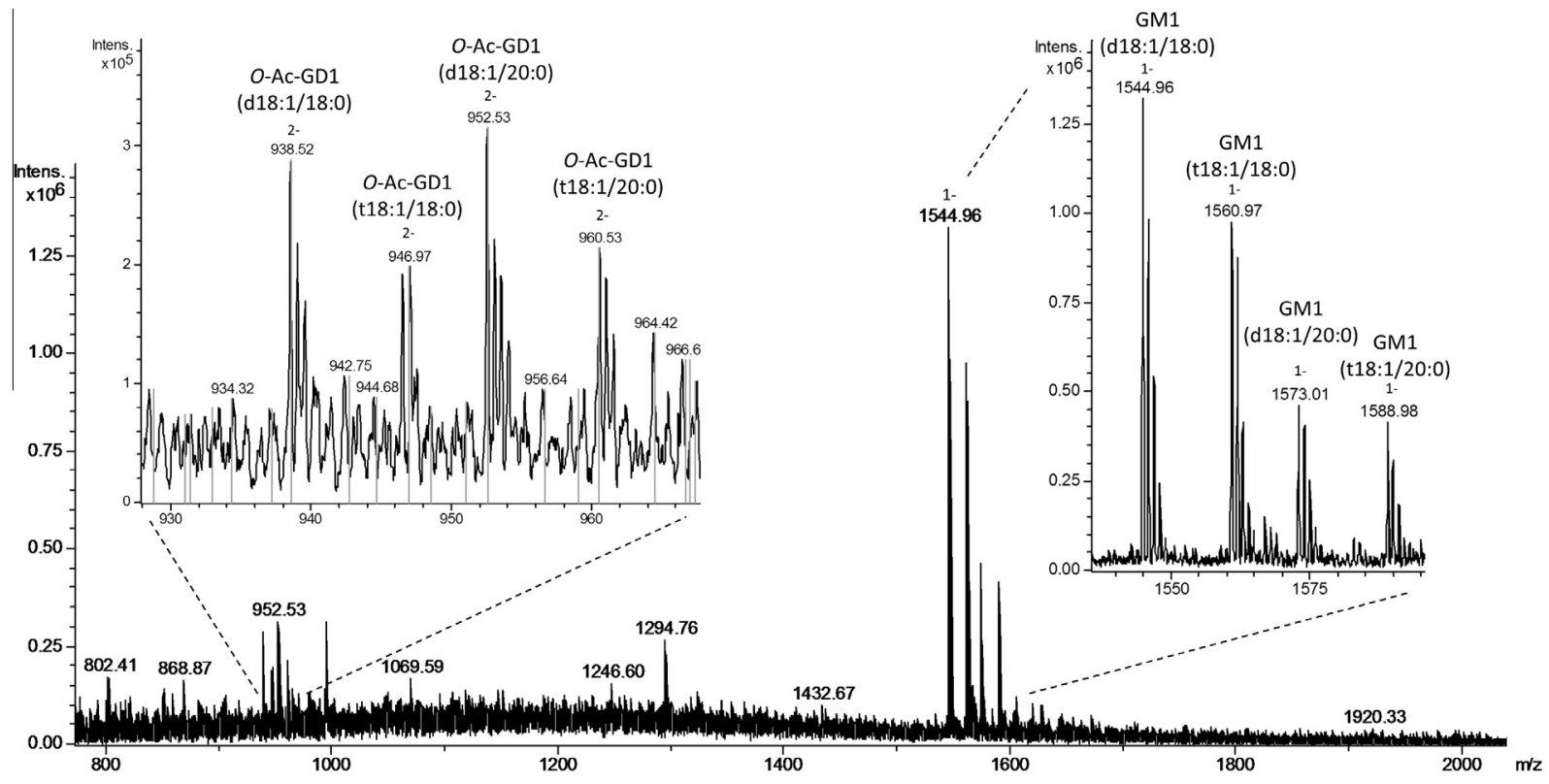

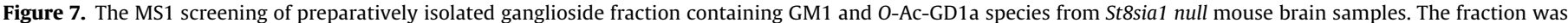
scrapped off the HPTLC plate and extracted from silica gel.

GD1 species. The doubly charged ion at $m / z 938.95$ corresponding to $O-A c-G D 1$ (d18:1/18:0) was subjected to tandem MS, and its product ion spectrum is presented in Figure 8. The major fragment ion relevant for deducing the presence of $O$-acetylation in the $O$-Ac-GD1 structure was detected at $\mathrm{m} / z 1586.9$ attributed to the sequence $\mathrm{O}$-Ac-NeuAc- $\mathrm{Gg}_{4} \mathrm{Cer}$ (Fig. 8) which is exactly for $\Delta m=291$ lower than the theoretical $m / z$ of the singly charged molecular ion corresponding to O-Ac-GD1 (d18:1/18:0), therefore indicating release of one NeuAc residue from the molecular ion, while $O$-Ac-NeuAc residue is still present within the fragment at $m / z$ 1586.9. The ion at $m / z 1544.92$ is for $\Delta m=42$ lower than the ion at $m / z 1586.9$, that confirms the release of the $O$-Ac residue. The other detected fragment ions, structurally defined in Figure 8, confirm the structure of $O$-Ac-GD1. Due to block in $b$-series ganglioside synthesis in this KO model, the presence of $b$-series isomer can be excluded, which is additionally supported by the absence of fragment ions at $m / z 599$ and 581 that would indicate the disialo-residue (Fig. 8). Therefore we can conclude that the ion at $\mathrm{m} / \mathrm{z}$ 938.95 corresponds to $O$-acetylated GD1a with d18:1/18:0 ceramide. The analogous fragmentation pattern was obtained by tandem MS of the ion at $m / z$ 952.92 that corresponds to O-Ac-GD1a (d18:1/20:2).

\section{Conclusions}

Brain gangliosides derived from mice with changed ganglioside composition and wild-type mice were characterized by means of HPTLC and mass spectrometry. Observed high structural complexity of ganglioside pattern was additionally diversified by sialic acid $O$-acetylation. The $O$-acetylated GD1a and $O$-acetylated GM3 


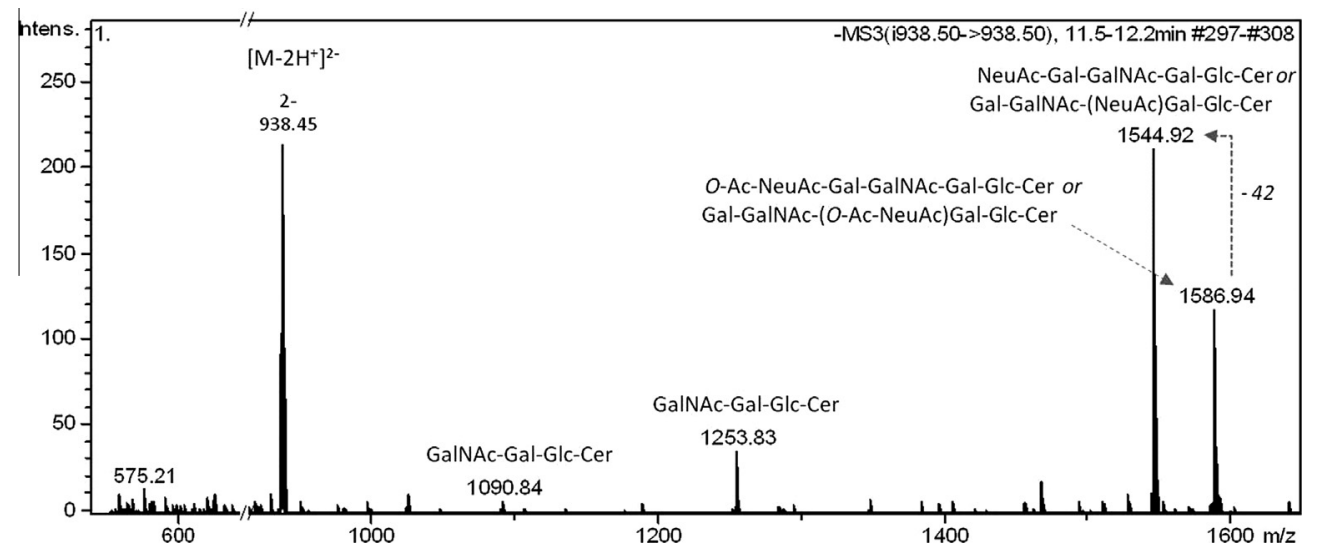

Figure 8. The product ion spectrum of the doubly charged molecular ion at $m / z 938.95$ corresponding to 0 -Ac-GD1a (d18:1/18:0).

species, not previously reported in a murine model with disrupted ganglioside biosynthesis, were identified. Additionally, detailed mass spectrometric structural analysis of brain ganglioside composition in St8sia1 null mice was provided. Our findings in mice with altered ganglioside biosynthesis indicate that the diversity of ganglioside structures, as well as acetylation patterns, is even higher than previously reported. These findings entail further investigation of newly detected $O$-acetylated structures, particularly their cellular localization and involvement in neurodevelopmental processes.

\section{Experimental}

\subsection{Mouse models with altered ganglioside biosynthesis}

Two genetically modified mouse models were used and compared to wt mice. Mice with disrupted St8sia1 gene (St8sia1 null) coding for $\alpha-N$-acetylneuraminate $\alpha-2,8$-sialyltransferase (GD3 synthase; EC 2.4.99.8) accumulate $a$-series gangliosides (GM1, GM2, GM3, GD1a) while they are depleted of both $b$ - and $c$-series gangliosides (GD3, GD1b, GT1b, GT3, GT2, GT1c) ${ }^{13,20}$ (Fig. 1). Mice with a disrupted B4galnt1 gene (B4galnt1null mice) which lack the enzyme GM2/GD2 synthase (UDP- $N$-acetyl-D-galactosamine:GM3/ GD3 $N$-acetyl-D- galactosaminyltransferase, EC 2.4.1.92) consequently do not synthesize the major gangliosides common to vertebrate brain but instead synthesize comparable amounts of simple gangliosides GM3 and GD3 ${ }^{11,21,22}$ (Fig. 1).

Adult St8sia1 null and B4galnt1 null mice were compared to ageand sex-matched wild-type littermates with the same genetic background (C57BL/6). Founder St8sia 1 null and B4galnt1 null mice (C57BL/6 background) originated from The Scripps Research Institute, La Jolla, California, as a part of the Consortium for Functional Glycomics. Sixteen mice were used in this study. Animals were kept in the same facility under same conditions in all aspects. All experimental procedures were approved by regional ethics committees for scientific experiments, and ethical guidelines were followed, in accordance with institutional and government guidelines.

\subsection{Ganglioside extraction and purification}

Mice were anesthetized with isofluorane and sacrificed. Brains were rapidly removed and dissected in a standardized manner. Frontal cortex tissue was weighed prior to ganglioside extraction procedure which was performed according to the method of Svennerholm and Fredman, ${ }^{35}$ as modified in our laboratory. ${ }^{36}$ Briefly, tissue samples were homogenized in ice-cold distilled water (W) in a Potter-Elvehjem glass-Teflon homogenizer to obtain $10 \%$ homogenate. Lipids were extracted twice using solvent mixture of chloroform (C):methanol (M) (1:2, by vol.), followed by partition and repartition by adding $\mathrm{M}$ and $\mathrm{W}$ to a final volume ratio 1:1:0.7. Upper phases were collected and evaporated to dryness. Dried ganglioside extracts were dissolved in $\mathrm{C} / \mathrm{M} / \mathrm{W}$ (60:30:4.5, by vol.) and left for $12 \mathrm{~h}$ at $+4{ }^{\circ} \mathrm{C}$ in order to induce precipitation of residual proteins. The precipitate was removed by centrifugation. The ganglioside extract was evaporated and the residue dissolved in $\mathrm{C} / \mathrm{M} /$ W (60:30:4.5, by vol.) and purified by gel filtration on SephadexG25 Fine gel (Sigma-Aldrich). The collected eluate was evaporated and purified ganglioside extracts were used for quantitative and qualitative analysis.

\subsection{Quantitative analysis of gangliosides}

Quantitative analysis of total ganglioside content was performed according to the spectrophotometric method of Svennerholm, ${ }^{37}$ as modified by Miettinen and Takki-Luukkainen. ${ }^{38}$ The absorbances of samples and $\mathrm{N}$-acetylneuraminic acid (Sigma-Aldrich) used as a standard in a range of known concentrations were determined at $580 \mathrm{~nm}$. The contents of ganglioside-bound sialic acid (GG-SA) were expressed as microgram GG-SA per gram of fresh tissue w.w.

\subsection{Qualitative analysis of native ganglioside mixtures and alkali-labile ganglioside species}

Qualitative analysis was performed by high performance thin layer chromatography (HPTLC) separation of individual ganglioside species on silica gel plate (Merck). HPTLC was used for three purposes: (1) collection of compositional data following resorcinol- $\mathrm{HCl}$ staining of separated ganglioside patterns, (2) investigation of alkali-labile ganglioside species and (3) preparative isolation of separated fractions for subsequent mass spectrometry (MS) analysis. In all cases plates were developed in a solvent system containing $\mathrm{C}, \mathrm{M}$, and $12 \mathrm{mM}$ aq $\mathrm{CaCl}_{2}$ (50:40:10, by vol.). The mixture of gangliosides from bovine brain (Cronassial, Fidia Research Laboratories) was used as a reference.

For qualitative analysis of native ganglioside mixtures the samples of purified ganglioside extracts were dissolved in an adequate volume of C:M:W (60:30:4.5, by vol.) and the aliquots corresponding to ganglioside content in $10 \mathrm{mg}$ of analyzed brain sample were spotted to the HPTLC plate. Resolved gangliosides were detected by resorcinol-HCl reagent. ${ }^{37}$ Images of the developed, stained plates were scanned and analyzed using Image analysis software (NIH) enabling relative quantification of individual ganglioside species, 
expressed as their relative proportions (\%) in total ganglioside content in the analyzed sample.

To check the presence of alkali-labile ganglioside species in purified ganglioside mixtures, alkaline treatment was performed according to the procedure of Sonnino et al. ${ }^{39}$ Briefly, dried aliquots corresponding to ganglioside content in $10 \mathrm{mg}$ of analyzed brain sample were dissolved in $100 \mu \mathrm{L}$ of $17 \mathrm{M}$ ammonia and incubated at room temperature overnight. After complete evaporation of ammonia, the sample was monitored by HPTLC as described above.

For preparative isolation of individually separated ganglioside fractions, native purified ganglioside mixtures were dissolved in an adequate volume of $\mathrm{C}: \mathrm{M}: \mathrm{W}$ (60:30:4.5, by vol.) and aliquots containing $15 \mu \mathrm{g}$ of GG-SA were spotted to the HPTLC plate. The plate was developed, dried, and the separated ganglioside fractions were visualized by iodine vapors. Ganglioside spots were marked with a pencil. After desorption of iodine, the marked areas were scrapped off the plate and the ganglioside fractions were extracted from silica gel in C:M:W (60:30:4.5, by vol.), filtrated on Celite (Serva) and evaporated for subsequent direct MS analysis.

\subsection{Mass spectrometry analysis}

MS and tandem MS experiments were performed on a Bruker amaZon ETD ion trap system (Bruker Daltonik GmbH, Germany) equipped with Apollo electrospray ionization source. The solutions of the native ganglioside mixtures were prepared by dissolving dried ganglioside extracts in methanol at concentrations of 1.6 $\mu \mathrm{M}$ GG-SA. The individual ganglioside fractions preparatively isolated from HPTLC plates were also dissolved in methanol. All samples were introduced into the electrospray ionization source by direct infusion at a flow rate of $65 \mu \mathrm{l} / \mathrm{h}$. The capillary voltage was set as $4500 \mathrm{~V}$ while high voltage end plate offset was $-500 \mathrm{~V}$. The temperature and flow rate of the drying gas were set at $210^{\circ} \mathrm{C}$ and $5 \mathrm{~L} / \mathrm{min}$, respectively. Helium was used as the collision gas. The CID excitation time was $40 \mathrm{~ms}$ and the amplitude was in the $0.4-1 \mathrm{~V}$ range. All spectra were acquired in negative ion mode using a scan range from 280 to $3000 \mathrm{~m} / \mathrm{z}$. DataAnalysis software 4.0 (Bruker Daltonik GmbH, Germany) was used for analysis (charge deconvolution and data reduction) and extraction of the MS and MS/MS data. Ganglioside ion identification was accomplished using our own software application 'GSL finder' and its accompanying ganglioside database.

\section{Acknowledgements}

These materials are based on work financed by Croatian Ministry of Science, Education and Sports (Grant Numbers: 108-1081870-1877, 108-1081870-2415, 219-0061194-2158, and 098-0982915-2945).

\section{Supplementary data}

Supplementary data associated with this article can be found, in the online version, at http://dx.doi.org/10.1016/j.carres.2013.09. 007.

\section{References}

1. Tettamanti, G.; Anastasia, L. Chemistry, Tissue and Cellular Distribution, and Developmental Profiles of Neural Sphingolipids. In Handbook of Neurochemistry and Molecular Neurobiology; Lajtha, A., Ed., 3rd ed.; Springer, 2010; pp 99-171.

2. Schnaar, R. L. FEBS Lett. 2010, 584, 1741-1747.

3. Fantini, J.; Barrantes, F. J. Biochim. Biophys. Acta 2009, 1788, 2345-2361.

4. Simons, K.; Ikonen, E. Nature 1997, 387, 569-572.

5. Fang, Y.; Wu, G.; Xie, X.; Lu, Z. H.; Ledeen, R. W. Neurochem. Res. 2000, 25, 931940.

6. Simon, B. M.; Malisan, F.; Testi, R.; Nicotera, P.; Leist, M. Cell Death Differ. 2002, 9, 758-767.

7. Yowler, B. C.: Schengrund, C. L. Glycoconj. J. 2004, 21, 287-293.

8. Lopez, P. H.; Schnaar, R. L. Curr. Opin. Struct. Biol. 2009, 19, 549-557.

9. Yu, R. K.; Tsai, Y. T.; Ariga, T.; Yanagisawa, M. J. Oleo Sci. 2011, 60, 537-544.

10. Yang, L. J.; Zeller, C. B.; Shaper, N. L.; Kiso, M.; Hasegawa, A.; Shapiro, R. E.; Schnaar, R. L. Proc. Natl. Acad. Sci. U.S.A. 1996, 93, 814-818.

11. Chiavegatto, S.; Sun, J.; Nelson, R. J.; Schnaar, R. L. Exp. Neurol. 2000, 166, 227 234.

12. Mitsuda, T.; Furukawa, K.; Fukumoto, S.; Miyazaki, H.; Urano, T.; Furukawa, K. J. Biol. Chem. 2002, 277, 11239-11246.

13. Handa, Y.; Ozaki, N.; Honda, T.; Furukawa, K.; Tomita, Y.; Inoue, M.; Furukawa, K.; Okada, M.; Sugiura, Y. Pain 2005, 117, 271-279.

14. Kittaka, D.; Itoh, M.; Ohmi, Y.; Kondo, Y.; Fukumoto, S.; Urano, T.; Tajima, O.; Furukawa, K.; Furukawa, K. Glycobiology 2008, 18, 509-516.

15. Posse de, C. E.; Sipione, S. FEBS Lett. 2010, 584, 1748-1759.

16. Furukawa, K.; Tokuda, N.; Okuda, T.; Tajima, O.; Furukawa, K. Semin. Cell Dev. Biol. 2004, 15, 389-396.

17. Proia, R. L. Philos. Trans. R. Soc. Lond. B Biol. Sci. 2003, 358, 879-883.

18. Yu, R. K.; Bieberich, E.; Xia, T.; Zeng, G. J. Lipid Res. 2004, 45, 783-793.

19. Sturgill, E. R.; Aoki, K.; Lopez, P. H.; Colacurcio, D.; Vajn, K.; Lorenzini, I.; Majic S.; Yang, W. H.; Heffer, M.; Tiemeyer, M.; Marth, J. D.; Schnaar, R. L. Glycobiology 2012, 22, 1289-1301.

20. Okada, M.; Itoh, M. M.; Haraguchi, M.; Okajima, T: Inoue, M.; Oishi, $\mathrm{H}_{\text {; }}$ Matsuda, Y.; Iwamoto, T.; Kawano, T.; Fukumoto, S.; Miyazaki, H.; Furukawa, K.; Aizawa, S.; Furukawa, K. J. Biol. Chem 2002, 277, 1633-1636.

21. Takamiya, K.; Yamamoto, A.; Furukawa, K.; Yamashiro, S.; Shin, M.; Okada, M.; Fukumoto, S.; Haraguchi, M.; Takeda, N.; Fujimura, K.; Sakae, M.; Kishikawa, M.; Shiku, H.; Furukawa, K.; Aizawa, S. Proc. Natl. Acad. Sci. U.S.A. 1996, 93, 10662-10667.

22. Sheikh, K. A.; Sun, J.; Liu, Y.; Kawai, H.; Crawford, T. O.; Proia, R. L.; Griffin, J. W.; Schnaar, R. L. Proc. Natl. Acad. Sci. U.S.A. 1999, 96, 7532-7537.

23. Matsuda, J.; Vanier, M. T.; Popa, I.; Portoukalian, J.; Suzuki, K. Proc. Jpn. Acad. Ser B-Phys. Biol. Sci. 2006, 82, 189-196.

24. Furukawa, K.; Aixinjueluo, W.; Kasama, T.; Ohkawa, Y.; Yoshihara, M.; Ohmi, Y.; Tajima, O.; Suzumura, A.; Kittaka, D.; Furukawa, K. J. Neurochem. 2008, 105, 1057-1066.

25. Siebert, H. C.; von Der Lieth, C. W.; Dong, X.; Reuter, G.; Schauer, R.; Gabius, H J.; Vliegenthart, J. F. Glycobiology 1996, 6, 561-572.

26. Schauer, R.; Schmid, H.; Pommerencke, J.; Iwersen, M.; Kohla, G. Adv. Exp. Med. Biol. 2001, 491, 325-342.

27. Varki, N. M.; Varki, A. Lab. Invest. 2007, 87, 851-857.

28. Mandal, C.; Schwartz-Albiez, R.; Vlasak, R. Top. Curr. Chem. 2012. http:// dx.doi.org/10.1007/128_2011_310 [Epub ahead of print].

29. Abad-Rodriguez, J.; Vallejo-Cremades, M.; Nieto-Sampedro, M. Glia 1998, 23 $156-168$.

30. Kohla, G.; Stockfleth, E.; Schauer, R. Neurochem. Res. 2002, 27, 583-592.

31. Negreiros, E. M.; Leao, A. C.; Santiago, M. F.; Mendez-Otero, R. J. Neurobiol. 2003, 57, 31-37.

32. Mendez-Otero, R.; Cavalcante, L. A. Neurosci. Lett. 1996, 204, 97-100.

33. Mendez-Otero, R.; Santiago, M. F. Braz. J. Med. Biol. Res. 2003, 36, 1003-1013.

34. Chen, H. Y.; Challa, A. K.; Varki, A. J. Biol. Chem. 2006, 281, 7825-7833.

35. Svennerholm, L.; Fredman, P. Biochim. Biophys. Acta 1980, 617, 97-109.

36. Vukelic, Z.; Metelmann, W.; Muthing, J.; Kos, M.; Peter-Katalinic, J. Biol. Chem 2001, 382, 259-274.

37. Svennerholm, L. Biochim. Biophys. Acta 1957, 24, 604-611.

38. Miettinen, T.; Takkiluukkainen, I. T. Acta Chem. Scand. 1959, 13, 856-858.

39. Sonnino, S.; Ghidoni, R.; Chigorno, V.; Masserini, M.; Tettamanti, G. Anal. Biochem. 1983, 128, 104-114. 